Original Research Paper

\title{
Distribution of Polyphenol Oxidase in some Cruciferae Vegetables
}

\author{
${ }^{1}$ Andi Nur Faidah Rahman, ${ }^{2}$ Februadi Bastian, ${ }^{3}$ Jumriah Langkong and ${ }^{4}$ Shuji Fujita \\ ${ }^{1,2,3}$ Department of Food Science and Technology, \\ Faculty of Agriculture, Hasanuddin University, Makassar 90245 Indonesia \\ ${ }^{4}$ Laboratory of Food Science, Faculty of Agriculture, Saga University, Saga 840-8502 Japan
}

Article history

Received: 02-02-2015

Revised: 11-09-2015

Accepted: 28-01-2016

Corresponding Author: Andi Nur Faidah Rahman Department of Food Science and Technology, Faculty of Agriculture, Hasanuddin

University, Makassar 90245

Indonesia

Email: faidah83@yahoo.com

\begin{abstract}
Enzymatic oxidation of polyphenolic compounds in damaged tissue of many fruits and vegetables induced undesirable browning as results in decreased marketability of products. A new type of polyphenol oxidase (PPO) that is "Phloroglucinol Oxidase $(\mathrm{PhO})$ " have been reported in turnip and cabbage, and the purified enzymes also had strong peroxidase (POD) activity. Distribution of PPO from different plant source of cruciferae vegetables were observed. Cabbage, broccoli, turnip and cauliflower PPOs from Indonesia and Japan strongly oxidized 1,3,5trihydroxybenzenes such as phloroglucinol (Phl) and phloroglucinol carboxylic acid, but not oxidized 1,2,3-trihydroxybenzenes and $O$ diphenol. On the other side, Chinese mustard, Chinese cabbage, shingensai, Japanese radish root, mizuna, takana, leaf mustard, katsuona and komatsuna also strongly oxidized 1,3,5-trihydroxybenzenes and little oxidized 1,2,3-trihydroxybenzenes such as pyrogallol and gallic acid, but not oxidized $o$-diphenol. These results indicated that the PPOs in all cruciferae vegetables are a group of a new type of PPO, however, the level activity of 7 varieties of vegetables from Indonesia and 15 varieties from Japan tested showed different activity level of PhO and POD.
\end{abstract}

Keywords: Cruciferae Vegetables, Polyphenol Oxidase, Peroxidase

\section{Introduction}

Cruciferae vegetables are vegetables of the family brassicaceae. These vegetables contain high phytochemicals compound such as polyphenols to prevent common human cancers. Oxidation of phenolic compounds by polyphenol oxidase (PPO) in the damaged tissue induced undesirable browning, resulting decrease the nutritional value of vegetables. Generally, PPO oxidized $o$-diphenols, such as found in Japanese butterbur (Han et al., 2009) and edible burdock (Han et al., 2006) strongly oxidized chlorogenic acid; edible yam (Fujita et al., 2006) strongly oxidized dopamine; broccoli florest (Gawlik-Dziki et al., 2007), mango (Wang et al., 2007) and mamey (Palma-Orozco et al., 2011) strongly oxidized catechol; garland chrysanthemum (Nkya et al., 2003) strongly oxidized catechol and epicatechin . On the other hand, the purified enzyme of soybean (Toiguchi et al., 1989) and one of the purified enzymes of edible burdock (Murao et al., 1993) not oxidized $o$-diphenols, but oxidized pyrogallol $(1,2,3-$ trihydroxybenzene) and phloroglucinol (1,3,5- trihydroxybenzene). In addition, a new type of PPO that is "Phloroglucinol Oxidase (PhO)" which only oxidizes 1,3,5-trihydroxybenzene such as phloroglucinol and phloroglucinol carboxylic acid was found in Satsuma mandarin (Fujita and Tono, 1980a) and cruciferae vegetables such as turnip (Fujita and Tono, 1980b) and cabbage (Fujita et al., $1995 ; 1997)$, and the purified enzymes also had strong POD activities. PPO and POD play roles in darkening of cruciferae vegetables, therefore, the experiments were performed to report the distributions of PPOs of cruciferae vegetables.

\section{Materials and Methods}

\section{Materials}

Variation of cruciferae vegetables were grown and purchased from a local market in Makassar, Indonesia (Table 1) and Saga, Japan (Table 2). Reagents used were obtained from Chemical Market in Makassar, Indonesia and from Wako Pure Chemical Co., Osaka, Japan. 


\section{Enzyme Extraction}

The fresh (fresh weights $500 \mathrm{~g}$ ) of various cruciferae vegetables were stirred with $0.1 \mathrm{M}$ phosphate buffer $(\mathrm{pH}$ $7.0)$ at $5^{\circ} \mathrm{C}$. The filtrate then centrifuged at $10,300 \times \mathrm{g}$ for $20 \mathrm{~min}$ at $5^{\circ} \mathrm{C}$ and the supernatant was salted out to $80 \%$ saturation with ammonium sulfate for $24 \mathrm{~h}$. The precipitated protein was collected by centrifugation $(10,300 \times \mathrm{g})$ and dissolved in a small quantity of $0.1 \mathrm{M}$ phosphate buffer ( $\mathrm{pH}$ 7.0) then dialyzed against the same buffer for $36 \mathrm{~h}$ at $5^{\circ} \mathrm{C}$ with four times changed of buffer and centrifuged again at the same condition. The supernatant was used as the enzyme solution.

\section{Assay of Enzyme Activity}

\section{PhO Activity}

$\mathrm{PhO}$ activity was determined by a spectrophotometric method (Fujita et al., 1993). The reaction mixture contained $0.5 \mathrm{~mL}$ of $20 \mathrm{mM}$ aqueous solution of phloroglucinol, $1.4 \mathrm{~mL}$ of $0.1 \mathrm{M}$ potassium phosphate $/ 0.1$ $\mathrm{M}$ sodium hydrogen phosphate buffer $(\mathrm{pH} 7.0)$ and 0.1 $\mathrm{mL}$ of the enzyme solution: it was incubated at $30^{\circ} \mathrm{C}$, after $10 \mathrm{~min}$ of incubation, $0.5 \mathrm{~mL}$ was taken out and added to $4.5 \mathrm{~mL}$ of distilled water and then measured immediately at $272 \mathrm{~nm}$ against an enzyme blank. One unit of enzyme activity was expressed as $\triangle \mathrm{A} 272$ of 0.1 per min and per $\mathrm{mL}$ of the enzyme solution (1.0 cm light path).

\section{PPO Activity}

PPO activity was measured by a colorimetric method (Yang et al., 2000). The mixture to be tested consisted of $0.5 \mathrm{~mL}$ of $10 \mathrm{mM}$ aqueous solution of various polyphenols, $4.0 \mathrm{~mL}$ of $0.1 \mathrm{M}$ phosphate buffer $(\mathrm{pH} 7.0)$ and $0.5 \mathrm{~mL}$ of the enzyme solution: it was incubated at $30^{\circ} \mathrm{C}$ for $5 \mathrm{~min}$ and reaction mixture was measured as absorbance at $420 \mathrm{~nm}(\triangle \mathrm{A} 420)$. One unit of enzyme activity was expressed as $\triangle \mathrm{A} 420$ of 0.1 per min and per $\mathrm{mL}$ of the enzyme solution (1.0 cm light path).

\section{POD Activity}

POD activity was measured by a colorimetric method (Fujita et al., 1995). The reaction mixture contained 0.5 $\mathrm{mL}$ of $0.1 \mathrm{M}$ aqueous solution of guaiacol, $4.1 \mathrm{~mL}$ of 0.1 $\mathrm{M}$ phosphate buffer ( $\mathrm{pH} 6.0$ ), $0.2 \mathrm{~mL}$ of $0.1 \%$ hydrogen peroxide and $0.2 \mathrm{~mL}$ of the enzyme solution: it was incubated at $30^{\circ} \mathrm{C}$ for $2 \mathrm{~min}$ and reaction mixture was measured as absorbance at $470 \mathrm{~nm}(\triangle \mathrm{A} 470)$. One unit of enzyme activity was defined as a change in absorbance of the mixture at $470 \mathrm{~nm}(\triangle \mathrm{A} 470)$ of $0.1 \mathrm{per}$ min and per $\mathrm{mL}$ enzyme solution $(1.0 \mathrm{~cm}$ light path).

\section{Determination of Protein}

Protein was determined by the method of Hartree (1972) using Bovine Serum Albumin (BSA, fraction V; Katayama Chemical Company, Osaka, Japan) as standard. Protein was determined as absorbance at $280 \mathrm{~nm}$.

\section{Results and Discussion}

Polyphenol oxidase (PPO) from different plant source of cruciferae vegetables were extracted by $80 \%$ ammonium sulfate saturation. Table 1 and 2 showed substrate specificity of crude PPO from various Indonesia's and Japan's cruciferae vegetables. Generally, PPO oxidized $o$-diphenols, as listed in Table 3 , garland chrysanthemum (Nkya et al., 2003) strongly oxidized chlorogenic acid and epicatechin; Japanese butterbur (Han et al., 2009) and apple (Murata et al., 1992) strongly oxidized chlorogenic acid; banana pulp and peel (Yang et al., 2000; 2001) strongly oxidized dopamine; red Swiss chard leaves strongly oxidized L-DOPA (Gao et al., 2009); and broccoli florest (Gawlik-Dziki et al., 2007), butter lettuce (Gawlik-Dziki et al., 2008) and cherry pulp (Jia et al., 2011) strongly oxidized catechol. However, substrate specificity of crude PPO from various Indonesia's and Japan's cruciferae vegetables (Table 1 and 2) showed different activity, all the cruciferae vegetables PPOs tested did not show activity toward $o$-diphenol. From 7 varieties of Indonesia's vegetables tested showed cabbage, broccoli and cauliflower PPOs strongly oxidized 1,3,5trihydroxybenzenes such as phloroglucinol and phloroglucinol carboxylic acid, but not oxidized 1,2,3trihydroxybenzenes and $o$-diphenols. On the other hand, PPOs of Chinese mustard, Chinese cabbage, shingensai and Japanese radish root PPO also strongly oxidized 1,3,5-trihydroxybenzenes, but little oxidized 1,2,3trihydroxybenzenes such as pyrogallol. In addition, the PPOs from 15 varieties of Japan's cruciferae vegetables such as cabbage, turnip, broccoli and cauliflower PPOs also strongly oxidized 1,3,5-trihydroxybenzenes such as phloroglucinol and phloroglucinol carboxylic acid, but not oxidized 1,2,3-trihydroxybenzenes and $o$-diphenol. On the other hand, PPOs of mizuna, takana, leaf mustard, katsuona and komatsuna also strongly oxidized 1,3,5trihydroxybenzenes, but have small activity toward pyrogallol. In addition, Japanese radish root PPO has small activity toward pyrogallol and gallic acid (Table 2). The results indicated that the PPOs from different plant source of cruciferae vegetables are a group of a new type of PPO that is "Phloroglucinol Oxidase (PhO)".

Distribution of PPO in the cruciferae vegetables from Indonesia and Japan showed in Table 4 and 5. The enzyme showed dual activities of phloroglucinol oxidase $(\mathrm{PhO})$ and peroxidase (POD) and it showed wide variation in $\mathrm{PhO}$ and $\mathrm{POD}$ activities. The level activities of $\mathrm{PhO}$ from 7 varieties of vegetables from Indonesia (Table 4) in order as follows (unit/mg protein): Japanese radish root (22.0) $>$ cauliflower $(20.7)>$ broccoli $(14.4)>$ cabbage (13.6) $>$ shingensai $(1.70)>$ Chinese mustard $(0.64)>$ Chinese cabbage (0.14). In addition, the level activities of POD in order as follows (unit/mg protein): Cabbage (60.0) $>$ Japanese radish root $(52.0)>$ broccoli (41.0) > 
cauliflower (40.0) $>$ shingensai $(9.0)>$ Chinese mustard (5.8) $>$ Chinese cabbage (3.4). On the other side, the level activity of $\mathrm{PhO}$ from 15 varieties of vegetables from Japan (Table 5) in order as follows (unit/mg protein): Japanese radish root $(127.0)>$ turnip $(96.8)>$ cabbage $(37.0)>$ broccoli $(36.0)>$ cauliflower $(16.8)>$ broccoli sprout $(5.4)$ $>$ nabana (4.6) $>$ Chinese cabbage (3.1) $>$ shingensai (2.7) $>$ daikon sprout $(2.0)>$ mizuna $(1.9)>$ takana $(1.1)>$ komatsuna $(1.0)>$ Chinese mustard $(0.9)>$ katsuona (0.3). On the other hand, the level activities of POD in order as follows (unit/mg protein): Turnip (432.0) > cabbage (292.0) $>$ Japanese radish root $(216.0)>$ broccoli (45.0) $>$ cauliflower $(41.3)>$ nabana $(26.5)>$ broccoli sprout $(19.3)>$ mizuna $(10.5)>$ daikon sprout $(9.5)>$ Chinese cabbage $(9.4)>$ shingensai $(9.0)>$ takana $(7.8)>$ Chinese mustard (5.8) $>$ komatsuna (5.2) $>$ katsuona (1.7).

Table 1. Substrate specificity of crude PPO from various Indonesia'scruciferae vegetables

\begin{tabular}{|c|c|c|c|c|c|c|c|}
\hline & Cabbage & Broccoli & $\begin{array}{l}\text { Chinese } \\
\text { mustard }\end{array}$ & $\begin{array}{l}\text { Chinese } \\
\text { Cabbage }\end{array}$ & Shingensai & Cauliflower & $\begin{array}{l}\text { Japanese } \\
\text { radish root }\end{array}$ \\
\hline $\begin{array}{l}\text { 1,3,5-trihydroxybenzenes: } \\
\text { phloroglucinol }\end{array}$ & $100 \pm 0.0$ & $100 \pm 0.0$ & $100 \pm 0.0$ & $100 \pm 0.0$ & $100 \pm 0.0$ & $100 \pm 0.0$ & $100 \pm 0.0$ \\
\hline $\begin{array}{l}\text { Phloroglucinol carboxylic acid } \\
\text { 1,2,3-trihydroxybenzenes: }\end{array}$ & $64 \pm 2.0$ & $71 \pm 5.0$ & $20 \pm 1.0$ & $15 \pm 1.0$ & $20 \pm 2.0$ & $31 \pm 2.0$ & $36 \pm 1.0$ \\
\hline Pyrogallol & $0 \pm 0.0$ & $0 \pm 0.0$ & $14 \pm 2.0$ & $11 \pm 1.0$ & $20 \pm 2.0$ & $0 \pm 0.0$ & $2.0 \pm 0.5$ \\
\hline Gallic acid & $0 \pm 0.0$ & $0 \pm 0.0$ & $0 \pm 0.0$ & $0 \pm 0.0$ & $0 \pm 0.0$ & $0 \pm 0.0$ & $0 \pm 0.0$ \\
\hline $\begin{array}{l}\text { o-diphenols: } \\
\text { catechol }\end{array}$ & $0 \pm 0.0$ & $0 \pm 0.0$ & $0 \pm 0.0$ & $0 \pm 0.0$ & $0 \pm 0.0$ & $0 \pm 0.0$ & $0 \pm 0.0$ \\
\hline Chlorogenic acid & $0 \pm 0.0$ & $0 \pm 0.0$ & $0 \pm 0.0$ & $0 \pm 0.0$ & $0 \pm 0.0$ & $0 \pm 0.0$ & $0 \pm 0.0$ \\
\hline DL-DOPA & $0 \pm 0.0$ & $0 \pm 0.0$ & $0 \pm 0.0$ & $0 \pm 0.0$ & $0 \pm 0.0$ & $0 \pm 0.0$ & $0 \pm 0.0$ \\
\hline Dopamine & $0 \pm 0.0$ & $0 \pm 0.0$ & $0 \pm 0.0$ & $0 \pm 0.0$ & $0 \pm 0.0$ & $0 \pm 0.0$ & $0 \pm 0.0$ \\
\hline$m$-diphenols resorcinol & $0 \pm 0.0$ & $0 \pm 0.0$ & $0 \pm 0.0$ & $0 \pm 0.0$ & $0 \pm 0.0$ & $0 \pm 0.0$ & $0 \pm 0.0$ \\
\hline
\end{tabular}

Above data were averages of three trials

Table 2. Substrate specificity of crude PPO from various Japan'scruciferae vegetables

\begin{tabular}{|c|c|c|c|c|c|c|c|c|c|c|}
\hline \multirow[b]{2}{*}{ Substrates } & \multicolumn{9}{|c|}{ Relative activity (\%) } & \multirow[b]{2}{*}{$\begin{array}{l}\text { Japanese } \\
\text { radish root }\end{array}$} \\
\hline & Mizuna & Takana & Cabbage & Turnip & Broccoli & $\begin{array}{l}\text { Leaf } \\
\text { mustard }\end{array}$ & Katsuona & Komatsuna & Cauliflower & \\
\hline $\begin{array}{l}\text { 1,3,5-trihydroxybenzenes: } \\
\text { phloroglucinol }\end{array}$ & $100 \pm 0.0$ & $100 \pm 0.0$ & $100 \pm 0.0$ & $100 \pm 0.0$ & $100 \pm 0.0$ & $100 \pm 0.0$ & $100 \pm 0.0$ & $100 \pm 0.0$ & $100 \pm 0.0$ & $100 \pm 0.0$ \\
\hline $\begin{array}{l}\text { phloroglucinol carboxylic acid } \\
\text { 1,2,3-trihydroxybenzenes: }\end{array}$ & $45 \pm 3.0$ & $99 \pm 8.0$ & $71 \pm 7.0$ & $47 \pm 5.0$ & $86 \pm 2.0$ & $34 \pm 2.0$ & $25 \pm 2.0$ & $40 \pm 4.1$ & $42 \pm 3.0$ & $46 \pm 5.2$ \\
\hline pyrogallol & $15 \pm 1.0$ & $18 \pm 2.0$ & $0 \pm 0.0$ & $0 \pm 0.0$ & $0 \pm 0.0$ & $4 \pm 0.5$ & $2 \pm 0.2$ & $26 \pm 2.0$ & $0 \pm 0.0$ & $2.5 \pm 0.2$ \\
\hline gallic acid & $0 \pm 0.0$ & $0 \pm 0.0$ & $0 \pm 0.0$ & $0 \pm 0.0$ & $0 \pm 0.0$ & $0 \pm 0.0$ & $0 \pm 0.0$ & $0 \pm 0.0$ & $0 \pm 0.0$ & $0.4 \pm 0.1$ \\
\hline o-diphenols: & & & & & & & & & & \\
\hline catechol & $0 \pm 0.0$ & $0 \pm 0.0$ & $0 \pm 0.0$ & $0 \pm 0.0$ & $0 \pm 0.0$ & $0 \pm 0.0$ & $0 \pm 0.0$ & $0 \pm 0.0$ & $0 \pm 0.0$ & $0 \pm 0.0$ \\
\hline chlorogenic acid & $0 \pm 0.0$ & $0 \pm 0.0$ & $0 \pm 0.0$ & $0 \pm 0.0$ & $0 \pm 0.0$ & $0 \pm 0.0$ & $0 \pm 0.0$ & $0 \pm 0.0$ & $0 \pm 0.0$ & $0 \pm 0.0$ \\
\hline DL-DOPA & $0 \pm 0.0$ & $0 \pm 0.0$ & $0 \pm 0.0$ & $0 \pm 0.0$ & $0 \pm 0.0$ & $0 \pm 0.0$ & $0 \pm 0.0$ & $0 \pm 0.0$ & $0 \pm 0.0$ & $0 \pm 0.0$ \\
\hline dopamine & $0 \pm 0.0$ & $0 \pm 0.0$ & $0 \pm 0.0$ & $0 \pm 0.0$ & $0 \pm 0.0$ & $0 \pm 0.0$ & $0 \pm 0.0$ & $0 \pm 0.0$ & $0 \pm 0.0$ & $0 \pm 0.0$ \\
\hline$m$-diphenols resorcinol & $0 \pm 0.0$ & $0 \pm 0.0$ & $0 \pm 0.0$ & $0 \pm 0.0$ & $0 \pm 0.0$ & $0 \pm 0.0$ & $0 \pm 0.0$ & $0 \pm 0.0$ & $0 \pm 0.0$ & $0 \pm 0.0$ \\
\hline
\end{tabular}

Above data were averages of three trials

Table 3. Substrate specificity of PPO from fruits and vegetables

\begin{tabular}{|c|c|c|c|c|c|c|c|c|c|}
\hline \multirow[b]{2}{*}{ Substrates } & \multicolumn{7}{|c|}{ Relative activity (\%) } & \multirow[b]{2}{*}{$\begin{array}{l}\text { Red Swiss } \\
\text { chard leaves } \\
\text { (Gao et al., } \\
2009)\end{array}$} & \multirow[b]{2}{*}{$\begin{array}{l}\text { Cherry } \\
\text { pulp } \\
\text { (Jia et al., } \\
2011 \text { ) }\end{array}$} \\
\hline & $\begin{array}{l}\text { Garland } \\
\text { chrysanthemum } \\
\text { (Nkya et al., } \\
2003 \text { ) }\end{array}$ & $\begin{array}{l}\text { Banana } \\
\text { pulp } \\
\text { (Yang et al., } \\
\text { 2000) }\end{array}$ & $\begin{array}{l}\text { Banana } \\
\text { peel } \\
\text { (Yang et al., } \\
2001) \\
\end{array}$ & $\begin{array}{l}\text { Broccoli } \\
\text { florest } \\
\text { (Gawlik-Dziki et al., } \\
\text { 2007) }\end{array}$ & $\begin{array}{l}\text { Butter } \\
\text { lettuce } \\
\text { (Gawlik-Dziki et al., } \\
2008 \text { ) }\end{array}$ & $\begin{array}{l}\text { Japanese } \\
\text { butterbur } \\
\text { (Han et al., } \\
\text { 2009) }\end{array}$ & $\begin{array}{l}\text { Apple } \\
\text { (Murata et al., } \\
\text { 1992) }\end{array}$ & & \\
\hline \multicolumn{10}{|l|}{ o-diphenols } \\
\hline catechol & 76 & 54 & 34 & 100 & 100 & 39 & & 86 & 100 \\
\hline 4-methylcatechol & & & & 62.76 & 88.5 & & & & \\
\hline Chlorogenic acid & 100 & 24.5 & 5.3 & 1.89 & 53.4 & 100 & 100 & 80 & \\
\hline DL-DOPA & 72 & 12.3 & 8 & & & 2 & 9.5 & & \\
\hline L-DOPA & & & & & & & & 100 & \\
\hline dopamine & 74 & 100 & 100 & & & 9 & 10.2 & & \\
\hline resorcinol & 0 & 0 & 0 & & & 0 & 0 & & 0 \\
\hline Caffeic acid & & 2 & 0.7 & 5.92 & 2.3 & 42 & & & \\
\hline D-catechin & 70 & 35.6 & 11.5 & & & 37 & 12.9 & & \\
\hline Epicatechin & 100 & 22.7 & 9.3 & & & 94 & 14.4 & & \\
\hline$p$-dimethylphenol & & & & & & & & 10 & \\
\hline$m$-dimethylphenol & & & & & & & & 11 & \\
\hline Ferulic acid & & & & 1.1 & 0.2 & & & & \\
\hline \multicolumn{10}{|l|}{ 1,2,3-trihydroxybenzenes: } \\
\hline Pyrogallol & 70 & 5.5 & 1.4 & & & 0 & 11.9 & & \\
\hline Gallic acid & 72 & 0 & 0 & & & 0 & & 73 & 70.1 \\
\hline \multicolumn{10}{|l|}{ 1,3,5-trihydroxybenzenes: } \\
\hline Phloroglucinol & 0 & 0 & 0 & 0.16 & & 0 & 0 & & 0 \\
\hline
\end{tabular}


Table 4. Distribution of PhO and POD in the cruciferae vegetables from Indonesia

\begin{tabular}{lll}
\hline & Specific activity (unit/mg protein) \\
Various of cruciferae vegetables & PhO & POD \\
\hline Chinese cabbage (Brassica campestris L.) & $0.14 \pm 0.01$ & $3.4 \pm 0.01$ \\
Chinese mustard (Brassica campestris L.) & $0.64 \pm 0.02$ & $5.8 \pm 0.01$ \\
Shingensai (Brassica rapa L. (Chinensis Group)) & $1.70 \pm 0.10$ & $9.0 \pm 1.00$ \\
Cauliflower (Brassica oleracea L.) & $20.7 \pm 1.00$ & $40.0 \pm 2.00$ \\
Broccoli (Brassica oleracea L.) & $14.4 \pm 1.10$ & $41.0 \pm 4.00$ \\
Japanese radish root (Raphanussativus L.) & $22.0 \pm 3.00$ & $52.0 \pm 6.00$ \\
Cabbage (Brassica oleracea L.) & $13.6 \pm 0.51$ & $60.0 \pm 8.00$ \\
\hline Above
\end{tabular}

Above data were averages of three trials

Table 5. Distribution of PhO and POD in the cruciferae vegetables from Japan

\begin{tabular}{|c|c|c|}
\hline \multirow[b]{2}{*}{ Various of cruciferae vegetables } & \multicolumn{2}{|c|}{ Specific activity (unit/mg protein) } \\
\hline & $\mathrm{PhO}$ & POD \\
\hline Komatsuna (Brassica rapa var. perviridis) & $1.0 \pm 0.1$ & $5.2 \pm 0.5$ \\
\hline Mizuna (Brassica rapa var. nipposinica) & $1.9 \pm 0.1$ & $10.5 \pm 1.0$ \\
\hline Takana (Brassica juncea Czern. et Coss. (Integlifolia Group)) & $1.1 \pm 0.2$ & $7.8 \pm 1.2$ \\
\hline Turnip (Brassica compestris L.) & $96.8 \pm 12.0$ & $432.0 \pm 20.0$ \\
\hline Chinese cabbage (Brassica campestris L.) & $3.1 \pm 0.1$ & $9.4 \pm 1.2$ \\
\hline Nabana (Brassica napusL.) & $4.6 \pm 0.5$ & $26.5 \pm 2.0$ \\
\hline Chinese mustard (Brassica campestris L.) & $0.9 \pm 0.1$ & $5.8 \pm 1.2$ \\
\hline Shingensai (Brassica rapa L. (Chinensis Group)) & $2.7 \pm 0.5$ & $9.0 \pm 1.0$ \\
\hline Katsuona (Brassica junceaCzern.) & $0.3 \pm 0.1$ & $1.5 \pm 0.5$ \\
\hline Daikon sprout (Raphanussativus L.) & $2.0 \pm 0.4$ & $9.5 \pm 2.0$ \\
\hline Broccoli sprout (Brassica oleracea L.) & $5.4 \pm 1.0$ & $19.3 \pm 3.0$ \\
\hline Cauliflower (Brassica oleracea L.) & $16.8 \pm 3.0$ & $41.3 \pm 10.0$ \\
\hline Broccoli (Brassica oleracea L.) & $36.0 \pm 7.1$ & $45.0 \pm 6.0$ \\
\hline Japanese radish root (Raphanussativus L.) & $127.0 \pm 15.0$ & $216.0 \pm 19.0$ \\
\hline Cabbage (Brassica oleracea L.) & $37.0 \pm 2.0$ & $292.0 \pm 18.0$ \\
\hline
\end{tabular}

\section{Conclusion}

Distribution of PPO from different plant source of cruciferae vegetables were determined. The PPO from all various cruciferae vegetables tested showed a group of a new type of PPO that is "Phloroglucinol Oxidase (PhO)" which were strongly oxidized 1,3,5trihydroxybenzene such as phloroglucinol and phloroglucinol carboxylic acid, and the purified enzymes also had strong POD activities. However, the level activity of 7 varieties of vegetables from Indonesia and 15 varieties from Japan tested showed different activity level of PhO and POD.

\section{Acknowledgement}

I would like to thank Hasanuddin University Indonesia and Saga University Japan, for support and allowing us to work on the Laboratory.

\section{Funding Information}

The research for this paper was financially supported by the Directorate General for Higher Education (DIKTI).

\section{Author's Contributions}

Andi Nur Faidah Rahman: Participated in all experiments.

Februadi Bastian and Jumriah Langkong: Coordinated the mouse work

Shuji Fujita: Designed the research plan and organized the study.

\section{Ethics}

This article is original and contains unpublished material. The corresponding author confirms that all of the other authors have read and approved the manuscript and no ethical issues involved.

\section{References}

Fujita, S. and T. Tono, 1980a. Peroxidase activity of phloroglucinoloxidase from satsuma mandarin fruits and effect of metal ions on the enzyme activities. Nippon Nōgeikagaku Kaishi, 54: 201-208.

DOI: 10.1271/nogeikagaku1924.54.201

Fujita, S. and T. Tono, 1980b. Purification of phloroglucinoloxidase from turnip and its properties. Nippon Nōgeikagaku Kaishi, 54: 429-435. DOI: 10.1271/nogeikagaku1924.54.429 
Fujita, S., H. Kawahara, S. Nazamid and T. Tono, 1993. spectrophotometric determination of phloroglucinol oxidase activity based on difference spectra. Bull. Faculty Agric. Saga Univ., 74: 81-86.

Fujita, S., N. Saari, M. Maegawa, T. Tetsuka and N. Hayashi et al., 1995. Purification and properties of polyphenol oxidase from cabbage (Brassica oleracea L.). J. Agric. Food Chem., 43: 1138-1142. DOI: $10.1021 / \mathrm{jf00053a005}$

Fujita, S., N. Saari, M. Maegawa, T. Tetsuka and N. Hayashi et al., 1997. Isolation and characterization of two phloroglucinol oxidases from cabbage (Brassica oleracea L.). J. Agric. Food Chem., 45: 59-63. DOI: 10.1021/jf9601271

Fujita, S., Y.Z. Han, C. Kouno, T. Matsuo and M. Yamashita et al., 2006. Purification and characterization of polyphenol oxidase from edible yam (Dioscorea opposita Thunb.). Food Sci. Technol. Res., 12: 235-239. DOI: 10.3136/fstr.12.235

Gao, Z.J., XH. Han and X.G. Xiao, 2009. Purification and characterisation of polyphenol oxidase from red Swiss chard (Beta vulgaris subspecies cicla) leaves. Food Chem., 117: 342-348.

DOI: 10.1016/j.foodchem.2009.04.013

Gawlik-Dziki, U., U. Szymanowska and B. Baraniak, 2007. Characterization of polyphenol oxidase from broccoli (Brassica oleracea var. botrytis italica) florets. Food Chem., 105: 1047-1053. DOI: 10.1016/j.foodchem.2007.05.012

Gawlik-Dziki, U., U. Złotek and M. Świeca, 2008. Characterization of polyphenol oxidase from butter lettuce (Lactuca sativa var. capitata L.). Food Chem., 107: 129-135. DOI: 10.1016/j.foodchem.2007.07.068

Han, Y., F. Zhao, T. Ogawa, M. Ohta and S. Fujita, 2009. Purification and characterization of polyphenol oxidase from Japanese butterbur (Petasites japonicus). Food Preser. Sci., 35: 179-186.

Han, Y.Z., M. Ayumu, N. Eline, N. Hayashi and S. Fujita, 2006. Purification and characterization of chlorogenic acid oxidase from edible burdock (Arctium lappa L.). Food Preser. Sci., 32: 275-281. DOI: $10.5891 /$ jafps.32.6_275

Hartree, E.F., 1972. Determination of protein: A modification of Lowry method that gives a linear photometric response. Anal. Biochem., 48: 422-427. DOI: 10.1016/0003-2697(72)90094-2
Jia, G., W. Baogang, F. Xiaoyuan, T. Haoru and L. Wensheng et al., 2011. Partial properties of polyphenol oxidase in sour cherry (Prunus cerasus L. CV. CAB) pulp. World J. Agric. Sci., 7: 444-449.

Murao, S., H. Oyama, Y. Nomura, T. Tono and T. Shin, 1993. Purification and characterization of Arctium lappa L. (edible burdock) polyphenol oxidase. Biosci. Biotechnol. Biochem., 57: 177-180. DOI: $10.1271 / \mathrm{bbb} .57 .177$

Murata, M., C. Kurokami and S. Homma, 1992. Purification and some properties of chlorogenic acid oxidase from apple (Malus pumila). Biosci. Biotechnol. Biochem., 56: 1705-1710. DOI: $10.1271 / \mathrm{bbb} .56 .1705$

Nkya, E., C. Kouno, Y.J. Li, C.P. Yang and N. Hayashi et al., 2003. Purification and characterization of polyphenol oxidase from garland chrysanthemum (Chrysanthemum coronarium L.). J. Agric. Food Chem., 51: 5467-5471. DOI: 10.1021/jf0212542

Palma-Orozco, G., A. Ortiz-Moreno, L. DorantesÀlvarez, J.G. Sampedro and H. Nàjera, 2011. Purification and partial biochemical characterization of polyphenol oxidase from mamey (Pouteria sapota). Phytochemistry, 72: 82-88.

DOI: $10.1016 /$ j.phytochem.2010.10.011

Toiguchi, S., K. Hayashi, Y. Adachi, M. Motoki and K. Haraguchi, 1989. Purification and characterization of soybean oxidase. Nippon Shokuhin Kogyo Gakkaishi, 36: 597-602. DOI: 10.3136/nskkk1962.36.7_597

Wang, J., W. Jiang, B. Wang, S. Liu and Z. Gong et al., 2007. Partial properties of polyphenol oxidase in mango (Mangifera indica L. CV. "tainong") pulp. J. Food Biochem., 31: 45-55.

DOI: $10.1111 / \mathrm{j} .1745-4514.2007 .00097 . x$

Yang, C.P., S. Fujita, K. Kohno, A. Kusubayashi and M. Ashrafuzzaman et al., 2001. Partial purification and characterization of polyphenol oxidase from banana (Musa sapientum L.) peel. J. Agric. Food Chem., 49: 1146-1149. DOI: 10.1021/jf001051i

Yang, C.P., S. Fujita, M. Ashrafuzzaman, N. Nakamura and N. Hayashi, 2000. Purification and characterization of polyphenol oxidase from banana (Musa sapientum L.) pulp. J. Agric. Food Chem., 48: 2732-2735. DOI: 10.1021/jf991037+ 